\title{
Myofibroblasts are associated with the progression of scirrhous gastric carcinoma
}

\author{
YUHIKO FUYUHIRO $^{1}$, MASAKAZU YASHIRO ${ }^{1,2}$, SATORU NODA $^{1}$, SHINICHIRO KASHIWAGI ${ }^{1}$, \\ JUNKO MATSUOKA $^{1}$, YOSUKE DOI ${ }^{1}$, YUKIHIRO KATO ${ }^{1}$, KAZUYA MUGURUMA ${ }^{1}$, \\ TETSUJI SAWADA $^{1}$ and KOSEI HIRAKAWA ${ }^{1}$ \\ ${ }^{1}$ Department of Surgical Oncology, and ${ }^{2}$ Oncology Institute of Geriatrics and Medical Science, \\ Osaka City University Graduate School of Medicine, Osaka, Japan
}

Received April 8, 2010; Accepted May 31, 2010

DOI: 10.3892/etm_00000086

\begin{abstract}
Fibroblasts, particularly myofibroblasts, affect the malignant progression of cancer cells in vitro. However, to date few reports have addressed the clinical significance of myofibroblasts in the gastric cancer microenvironment. This study examined the correlation between myofibroblast expression and clinicopathological features in gastric carcinoma. A total of 265 primary gastric tumors resected by gastrectomy were stained with antibodies against $\alpha$-smooth muscle actin and vimentin. Stromal cells positive for vimentin were considered to be fibroblasts. Myofibroblasts were defined as fibroblasts positive for $\alpha$-smooth muscle staining. Myofibroblast-positive gastric carcinoma was established when myofibroblasts accounted for more than $25 \%$ of fibroblasts in the cancer stroma. Myofibroblast expression was positive in 92 (35\%) of the 265 gastric carcinomas. Myofibroblast expression showed a significantly $(\mathrm{p}<0.001)$ high frequency in advanced gastric cancers (76 of 146), in comparison to the early stage cancers (16 of 119). Taken together, there was a statistically significant correlation between myofibroblast expression and scirrhous type gastric cancer $(\mathrm{p}<0.001)$, lymph node metastasis $(\mathrm{p}<0.001)$, lymphatic invasion $(\mathrm{p}<0.001)$ and peritoneal dissemination $(\mathrm{p}=0.005)$. The prognosis of patients with tumors positive for myofibroblast expression was significantly $(\mathrm{p}<0.001)$ worse, while a multivariate analysis revealed that myofibroblast expression was not an independent prognostic factor. These findings suggest that myofibroblasts are associated with scirrhous gastric cancer. Overexpression of myofibroblasts may therefore be a useful prognostic indicator of gastric carcinoma.
\end{abstract}

Correspondence to: Dr Masakazu Yashiro, Department of Surgical Oncology, Osaka City University Graduate School of Medicine, 1-4-3 Asahi-machi, Abeno-ku, Osaka 545-8585, Japan

E-mail: m9312510@med.osaka-cu.ac.jp

Key words: myofibroblasts, scirrhous gastric carcinoma

\section{Introduction}

There is increasing evidence that the stroma is involved in the growth and metastasis of several types of cancer, including gastric (1), breast $(2,3)$ and prostate (4) cancer. The stroma consists of a variety of components, including fibroblasts, macrophages, and the extracellular matrix $(5,6)$. Among these cells, fibroblasts constitute a major stromal compartment and play a critical role in the regulation of tumor growth. Among the various types of fibroblasts, myofibroblasts, which are distinct from normal fibroblasts in their expression of both vimentin and $\alpha$-smooth muscle actin ( $\alpha$-SMA), have recently been implicated to have important functions in epithelial solid tumor biology, such as neoplastic progression, tumor growth and metastasis in vitro (7-9). De Wever et al emphasized the myofibroblast as a driver of invasive cancer growth; the role of myofibroblasts in tissue development was also proposed (10).

Scirrhous gastric cancer cells proliferate and extensively invade the submucosa in the gastric submucosa accompanied by abundant fibrosis (11). Interactions have been reported to exist between scirrhous gastric cancer cells and orthotopic fibroblasts, thus suggesting that the proliferation of scirrhous gastric carcinoma is related to growth factor production by gastric fibroblasts (12). Myofibroblasts have also been shown to be present in gastric cancer, but their histogenesis remains unclear. Despite increasing reports illustrating the role of tumor-stroma in cancer progression, no consensus has been reached regarding whether myofibroblasts regulate tumor development positively or negatively. Few reports of clinical studies of scirrhous gastric cancer discuss the significance of myofibroblasts. Therefore, the present study was performed to investigate the significance of myofibroblast expression in gastric carcinomas.

\section{Materials and methods}

Clinical materials. A total of 265 patients who had undergone resection of a primary gastric tumor at our institute were enrolled in this study. Tumor specimens were fixed in $10 \%$ formaldehyde solution and embedded in paraffin. Sections $(4-\mu \mathrm{m})$ were cut and mounted on glass slides. The pathologic diagnoses and classifications were made according to the 


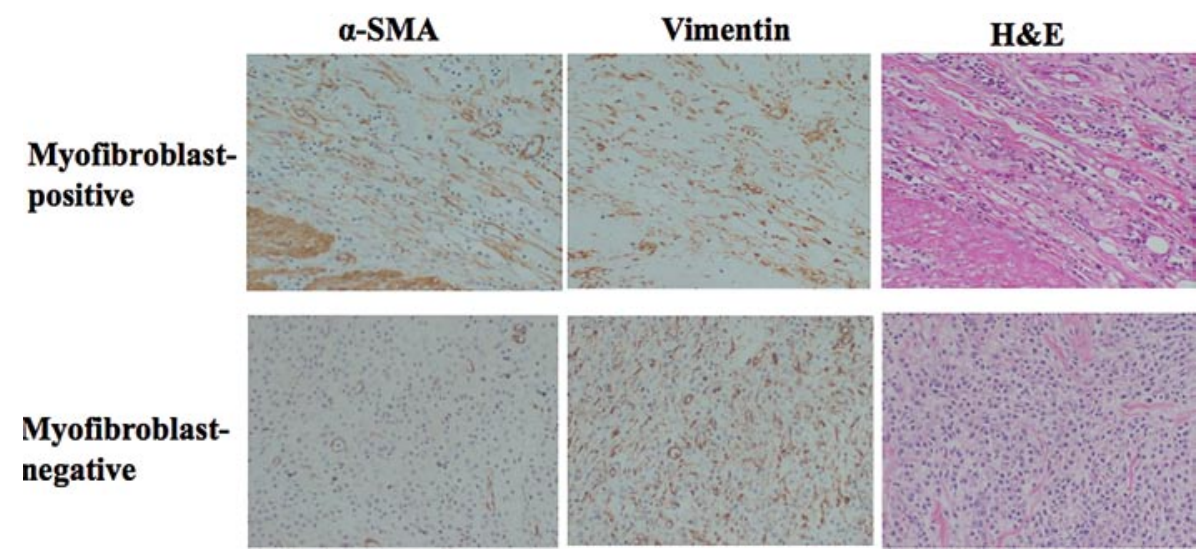

Figure 1. Myofibroblast expression in stromal cells. Expression of $\alpha$-smooth muscle actin was observed in the stroma in a diffuse-type carcinoma in original magnification, x200. Expression of vimentin was observed at the stroma.

Japanese Classification of Gastric Carcinoma (13). The median follow-up time for all 265 patients was 58 months (range, 1-177 months). The median follow-up time for the patients that succumbed to the disease was 25 months $(\mathrm{n}=88)$ compared with 75 months for surviving patients $(\mathrm{n}=177)$. Thirty-one patients were lost during more than 60 months of follow-up. Kaplan-Meier overall survival curves were calculated from the date of surgery.

Antibodies and reagents. A mouse monoclonal antibody which recognizes $\alpha$-SMA (clone 1A4) and a mouse monoclonal antibody which recognizes vimentin (clone Vim 3B4) were purchased from DakoCytomation (Cambridge, UK). Normal rabbit serum, normal mouse immunoglobulin G, biotinylated rabbit anti-mouse immunoglobulin $\mathrm{G}$, streptavidin-peroxidase reagent and diaminobenzidine were purchased from Nichirei Corp. (Tokyo, Japan).

Immunohistochemical techniques. Since there is no myofibroblast-specific immunocytochemical marker, characterization of human tumor-associated myofibroblasts is based on a combination of positive markers such as vimentin and $\alpha$-SMA. The methods for the immunohistochemical determination of $\alpha$-SMA and vimentin are described in detail in the manufacturer's instructions. Briefly, the slides were deparaffinized in xylene and hydrated in decreasing concentrations of ethyl alcohol. The tissues were heated for $20 \mathrm{~min}$ at $105^{\circ} \mathrm{C}$ and at $0.4 \mathrm{~kg} /$ $\mathrm{cm}^{2}$ by autoclave in Target Retrieval Solution (Dako Co., Carpinteria, CA). The sections were then dewaxed and incubated with $3 \%$ hydrogen peroxide $\mathrm{v} / \mathrm{v}$ in methanol for 15 min to block endogenous peroxidase activity. Next, the sections were washed in phosphate-buffered saline (PBS) and incubated in $10 \%$ normal rabbit serum $\mathrm{v} / \mathrm{v}$ for $10 \mathrm{~min}$ to reduce non-specific antibody binding. The specimens were incubated with $\alpha$-SMA antibodies (1:200) or vimentin antibodies (1:200) for $1 \mathrm{~h}$ at room temperature followed by three washes with PBS. Sections were incubated with biotinylated rabbit anti-mouse immunoglobulin $\mathrm{G}$ for $30 \mathrm{~min}$, followed by three washes with PBS. Slides were treated with streptavidin-peroxidase reagent for $15 \mathrm{~min}$ and washed with PBS three times. Finally, the slides were incubated in PBS diaminobenzidine and $1 \%$ hydrogen peroxide $\mathrm{v} / \mathrm{v}$ for $20 \mathrm{sec}$, counterstained with Mayer's hematoxylin and mounted.

Immunohistochemical determination of $\alpha$-smooth muscle actin and vimentin. The tumor specimens showed various staining patterns against the anti- $\alpha$-SMA and anti-vimentin antibodies. Vimentin-positive stromal cells were considered to be fibroblasts. Myofibroblasts were defined as fibroblasts which were positive for $\alpha$-SMA staining. Smooth muscle was defined as being $\alpha$-SMA-positive and vimentin-negative. The myofibroblast expression level was semi-quantitatively analyzed according to the percentage of fibroblasts showing $\alpha$-SMA positivity: $0,0 \% ; 1+, 1-24 \% ; 2+, 25-49 \% ; 3+, \geq 50 \%$. Myofibroblast expression was considered positive when scores were $\geq 2+$, and negative when scores were $\leq 1+$ (Fig. 1). The slides were interpreted by two investigators without knowledge of the corresponding clinicopathological data.

Statistical analysis. The $\chi^{2}$ test was used to determine the significance of the differences between the covariates. Survival durations were calculated using the Kaplan-Meier method and were analyzed by the log-rank test to compare the cumulative survival durations in the patient groups. The Cox proportional hazards model was used to compute univariate and multivariate hazards ratios for the study parameters. For all tests, a p-value $<0.05$ was defined as statistically significant. The SPSS software program (SPSS Japan, Tokyo, Japan) was used for the analyses.

\section{Results}

Correlation between the clinicopathological factors and myofibroblast expession. Myofibroblast expression was positive in $92(35 \%)$ of the 265 gastric carcinoma specimens, in $16(13 \%)$ of the 119 early stage gastric carcinoma specimens, and in $76(52 \%)$ of the 146 advanced gastric carcinoma specimens. The relationships between myofibroblast positivity and clinicopathological features of the tumors are shown in Table I. A significantly $(\mathrm{p}<0.001)$ high frequency of myofibroblast positivity was observed in the advanced gastric cancers (76 of 146) in comparison to the early stage cancers (16 of 119). Therefore, a statistically significant 
Table I. Correlation between clinicopathological factors and myofibroblast expression.

\begin{tabular}{|c|c|c|c|}
\hline \multirow[b]{2}{*}{$\begin{array}{l}\text { Clinicopathological } \\
\text { factors }\end{array}$} & \multicolumn{2}{|c|}{ Myofibroblast expression ${ }^{a}$} & \multirow[b]{2}{*}{ p-value } \\
\hline & $\begin{array}{c}\text { Positive } \\
\mathrm{n}=92(35 \%)\end{array}$ & $\begin{array}{c}\text { Negative } \\
\mathrm{n}=173(65 \%)\end{array}$ & \\
\hline \multicolumn{4}{|l|}{ Invasion depth } \\
\hline Early stage cancer & $16(13 \%)$ & $103(87 \%)$ & \\
\hline Advanced cancer & $76(52 \%)$ & $70(48 \%)$ & $<0.001$ \\
\hline \multicolumn{4}{|l|}{ Macroscopic type $^{b}$} \\
\hline Type $0,1,2,3$ & $65(29 \%)$ & $157(71 \%)$ & \\
\hline Type 4 (scirrhous type) & $27(63 \%)$ & $16(37 \%)$ & $<0.001$ \\
\hline \multicolumn{4}{|l|}{ Histological type } \\
\hline Differentiated & $34(30 \%)$ & $79(70 \%)$ & \\
\hline Undifferentiated & $58(38 \%)$ & $94(62 \%)$ & $0.172^{c}$ \\
\hline \multicolumn{4}{|l|}{ Peritoneal metastasis } \\
\hline Positive & $12(57 \%)$ & $9(43 \%)$ & \\
\hline Negative & $80(33 \%)$ & $164(67 \%)$ & 0.024 \\
\hline \multicolumn{4}{|l|}{ Venous invasion } \\
\hline Positive & $25(45 \%)$ & $31(55 \%)$ & \\
\hline Negative & $67(32 \%)$ & $142(68 \%)$ & 0.079 \\
\hline \multicolumn{4}{|l|}{ Lymph node metastasis } \\
\hline Positive & $57(51 \%)$ & $54(49 \%)$ & \\
\hline Negative & $35(23 \%)$ & $119(77 \%)$ & $<0.001$ \\
\hline \multicolumn{4}{|l|}{ Lymphatic invasion } \\
\hline Positive & $56(48 \%)$ & $60(52 \%)$ & \\
\hline Negative & $36(24 \%)$ & $113(76 \%)$ & $<0.001$ \\
\hline \multicolumn{4}{|l|}{ Cytology ${ }^{c}$} \\
\hline Positive & $19(56 \%)$ & $15(44 \%)$ & \\
\hline Negative & $73(32 \%)$ & $158(68 \%)$ & 0.005 \\
\hline
\end{tabular}

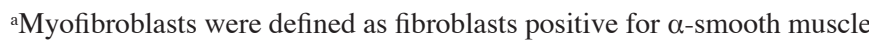

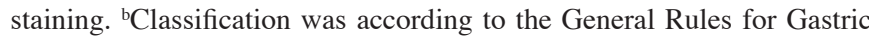
Cancer Study of the Japanese Research Society for Gastric Cancer. Type 0 is defined as superficial flat tumors with or without minimal elevation or depression. Type 1 is defined as a polypoid tumor, sharply demarcated from the surrounding mucosa and usually attached on a wide base. Type 2 is defined as polypoid tumor with ulceration and with sharply demarcated margins. Type 3 is defined as ulcerated carcinoma with cancer infiltration into the surrounding wall. Type 4 is defined as diffusely infiltrating flat carcinoma in which ulceration is usually not a marked feature. 'Cytology' is defined as peritoneal lavage cytology at laparotomy as a standard method for the detection of free tumor cells.

correlation was found between myofibroblast expression and scirrhous type gastric cancer $(\mathrm{p}<0.001)$. Myofibroblast positivity was also significantly present in patients with lymph node metastasis $(\mathrm{p}<0.001)$, positive cytology $(\mathrm{p}=0.005)$ and lymphatic invasion $(\mathrm{p}<0.001)$. 'Cytology' is defined as peritoneal lavage cytology at laparotomy as a standard method for the detection of free tumor cells. There was no statistically significant association between myofibroblast positivity and histological type, peritoneal dissemination and venous invasion.
Table II. Univariate analysis with respect to overall survival in gastric cancer.

\begin{tabular}{|c|c|c|c|}
\hline Parameter & Risk ratio & $95 \% \mathrm{CI}$ & $\mathrm{p}$-value \\
\hline \multicolumn{4}{|c|}{ Myofibroblast expression } \\
\hline Positive vs. negative & 1.482 & $1.146-1.917$ & 0.003 \\
\hline \multicolumn{4}{|l|}{ Invasion depth } \\
\hline $\begin{array}{l}\text { Early stage vs. } \\
\text { advanced cancer }\end{array}$ & 1.692 & $1.323-2.163$ & $<0.001$ \\
\hline \multicolumn{4}{|l|}{ Macroscopic type } \\
\hline $\begin{array}{l}\text { Type } 1,2,3 \text { vs. } \\
\text { Type } 4 \text { (scirrhous type }\end{array}$ & 3.366 & $2.389-4.742$ & $<0.001$ \\
\hline \multicolumn{4}{|l|}{ Histological type } \\
\hline Diffuse vs. intestinal & 1.084 & $0.848-1.386$ & 0.520 \\
\hline \multicolumn{4}{|l|}{ Peritoneal metastasis } \\
\hline Positive vs. negative & 6.462 & $3.926-10.638$ & $<0.001$ \\
\hline \multicolumn{4}{|l|}{ Cytology } \\
\hline Positive vs. negative & 4.417 & $3.002-6.501$ & $<0.001$ \\
\hline \multicolumn{4}{|l|}{ Venous invasion } \\
\hline Positive vs. negative & 1.363 & $1.011-1.838$ & 0.042 \\
\hline \multicolumn{4}{|l|}{ Lymph node metastasis } \\
\hline Positive vs. negative & 1.911 & $1.490-2.450$ & $<0.001$ \\
\hline \multicolumn{4}{|l|}{ Lymphatic invasion } \\
\hline Positive vs. negative & 1.663 & $1.299-2.130$ & $<0.001$ \\
\hline
\end{tabular}

Survival. The prognosis of patients with tumors positive for myofibroblast expression was significantly $(p<0.001)$ worse than that of patients with tumors negative for myofibroblast expression (Fig. 2A). The 5-year survival of the patients with myofibroblast-positive tumors was $57 \%$ in comparison to $79 \%$ for those patients with negative tumors. The prognosis of patients who underwent a curative resection (R0) with myofibroblast-positive tumors was significantly $(\mathrm{p}<0.0210)$ worse than the prognosis of patients with tumors negative for myofibloblast expression (Fig. 2B). Univariate analysis revealed that myofibroblast expression $(\mathrm{p}=0.003)$, advanced cancer $(\mathrm{p}<0.001)$, peritoneal dissemination $(\mathrm{p}<0.001)$, venous invasion $(\mathrm{p}=0.042)$ and lymph node metastasis $(\mathrm{p}<0.001)$ were significantly correlated with patient survival (Table II). Multivariate analysis indicated that the macroscopic type and peritoneal dissemination were independent prognostic factors (Table III), while myofibroblast expression $(p=0.290)$ was not an independent prognostic factor.

\section{Discussion}

The present study demonstrated that myofibroblast expression was significantly associated with advanced stage diffusetype gastric cancer, particularly scirrhous type and distant metastasis. In the present study, 43 cases of Type 4 showed diffusely infiltrating carcinoma accompanied by extensive 
A

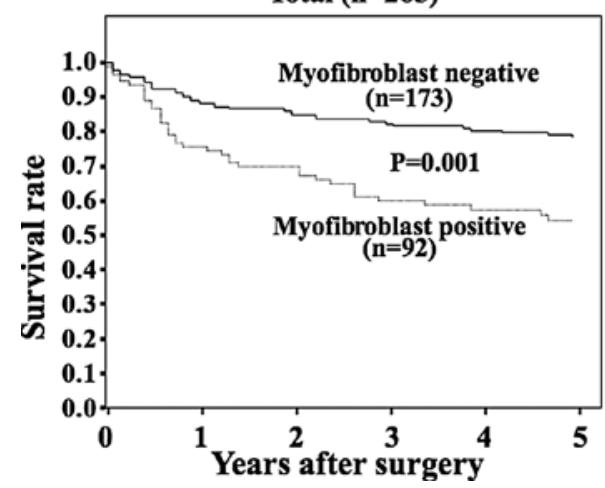

B

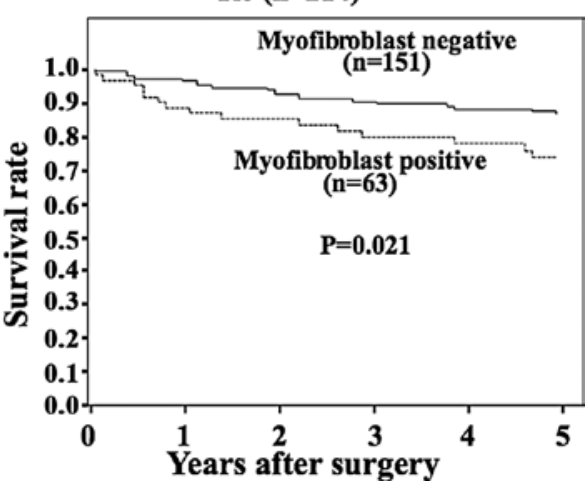

Figure 2. Patient survival. (A) Overall survival of the studied patients based on myofibroblast expression. (B) Overall survival of the patients with curative resection (R0) based on myofibroblast expression.

Table III. Multivariate analysis with respect to overall survival in gastric cancer.

\begin{tabular}{lccc}
\hline Parameter & Risk ratio & $95 \%$ CI & p-value \\
\hline $\begin{array}{l}\text { Myofibroblast expression } \\
\text { Positive vs. negative }\end{array}$ & 1.076 & $0.664-1.744$ & 0.766 \\
$\begin{array}{l}\text { Invasion depth } \\
\text { Early stage vs. } \\
\text { advanced cancer }\end{array}$ & 3.366 & $1.401-8.088$ & 0.007 \\
$\begin{array}{l}\text { Macroscopic type } \\
\text { Type 1, 2, 3 vs. }\end{array}$ & & & \\
Type 4 (scirrhous type) & 4.344 & $2.136-8.838$ & $<0.001$ \\
$\begin{array}{l}\text { Histological type } \\
\text { Diffuse vs. intestinal }\end{array}$ & 1.118 & $0.586-2.123$ & 0.735 \\
$\begin{array}{l}\text { Peritoneal metastasis } \\
\text { Positive vs. negative }\end{array}$ & 2.184 & $1.162-4.105$ & 0.015 \\
$\begin{array}{l}\text { Venous invasion } \\
\text { Positive vs. negative }\end{array}$ & 1.543 & $0.869-2.741$ & 0.139 \\
$\begin{array}{l}\text { Lymph node metastasis } \\
\text { Positive vs. negative }\end{array}$ & 1.308 & $0.675-2.533$ & 0.426 \\
\hline
\end{tabular}

CI, confidence interval.

stromal fibroblasts, which is distinguished as scirrhous gastric carcinoma (11). This type of carcinoma accounts for approximately $10 \%$ of all gastric carcinomas, and patients presenting with this type are associated with a poorer prognosis in comparison to other types of gastric carcinomas, thus reflecting a rapid proliferation of cancer cells (14). Interactions between scirrhous gastric cancer cells and orthotopic fibroblasts have been previously reported (12). Myofibroblasts, among orthotopic stromal cells, might thus play an important role in cancer progression in the development of scirrhous type gastric cancer. Orimo et al found that myofibroblasts exhibited significant positive signals of various growth factors in breast cancer $(9,15)$. Our previous study indicated that keratinocyte growth factor, transforming growth factor- $\beta$ and hepatocyte growth factor secreted by human gastric fibroblasts might stimulate proliferation and invasion of human scirrhous gastric carcinoma cells $(16,17)$. Myofibroblasts are thought to accelerate the aggressive phenotype of scirrhous gastric carcinoma via these growth factors.

The prognosis of patients with myofibroblast-positive tumors such as colorectal cancer (18) was reported to be significantly worse than the prognosis of patients with myofibroblast-negative tumors, thus suggesting that myofibroblast expression is indicative of high malignancy and might also be associated with diffusely invasive growth and poor prognosis. Overexpression of myofibroblasts might be a useful prognostic indicator, while multivariate analysis revealed that myofibroblast expression was not an independent prognostic factor.

Differentiation from resident stromal fibroblasts into myofibroblasts is induced by paracrine signals generated by repairing or inflamed tissues. Among these signals, TGF- $\beta$ is a wellknown inducer of myofibroblasts which stimulates fibroblasts to differentiate into myofibroblasts in cancer tissues $(2,19,20)$. Overexpression of TGF- $\beta$ is reported to accelerate metastasis and is thus correlated with the poor prognosis of gastric tumors, particularly for scirrhous gastric carcinoma $(21,22)$. Moreover, TGF- $\beta$ is produced to a greater extent by most scirrhous gastric cancer cells than by other types of gastric cancer cells (23). These findings suggest that myofibroblasts induced by TGF- $\beta$ from scirrhous gastric cancer cells might thus be responsible for the poor prognosis observed for scirrhous gastric cancer.

In conclusion, myofibroblasts in the microenvironment of gastric cancer were found to be significantly associated with an advanced stage, particularly for the macroscopically scirrhous type gastric carcinoma. Overexpression of myofibroblasts may therefore be a useful prognostic indicator for such cases.

\section{Acknowledgements}

We thank Masako Shinkawa (Osaka City University Graduate School of Medicine) for the technical advice on the immunohistochemical staining. This study was supported, in part, by Grants-in-Aid for Scientific Research (nos. 18591475, 20591073 and 18390369) from the Ministry of Education, Science, Sports, Culture and Technology of Japan. 


\section{References}

1. Nakayama H, Enzan H, Miyazaki E and Toi M: Alpha smooth muscle actin-positive stromal cells in gastric carcinoma. J Clin Pathol 55: 741-744, 2002.

2. Ronnov-Jessen L, Petersen OW, Koteliansky VE and Bissell MJ: The origin of the myofibroblasts in breast cancer. Recapitulation of tumor environment in culture unravels diversity and implicates converted fibroblasts and recruited smooth muscle cells. J Clin Invest 95: 859-873, 1995.

3. Chauhan H, Abraham A, Phillips JR, Pringle JH, Walker RA and Jones JL: There is more than one kind of myofibroblast: analysis of CD34 expression in benign, in situ, and invasive breast lesions. J Clin Pathol 56: 271-276, 2003.

4. Tuxhorn JA, McAlhany SJ, Dang TD, Ayala GE and Rowley DR: Stromal cells promote angiogenesis and growth of human prostate tumors in a differential reactive stroma (DRS) xenograft model. Cancer Res 62: 3298-3307, 2002.

5. Albini A and Sporn MB: The tumour microenvironment as a target for chemoprevention. Nat Rev Cancer 7: 139-147, 2007.

6. Barth PJ and Westhoff CC: CD34+ fibrocytes: morphology, histogenesis and function. Curr Stem Cell Res Ther 2: 221-227, 2007.

7. Eyden B: The myofibroblast: a study of normal, reactive and neoplastic tissues, with an emphasis on ultrastructure. J Submicrosc Cytol Pathol: 231-296, 2007.

8. Eyden B: The myofibroblast: phenotypic characterization as a prerequisite to understanding its functions in translational medicine. J Cell Mol Med 12: 22-37, 2008.

9. Orimo A, Gupta PB, Sgroi DC, et al: Stromal fibroblasts present in invasive human breast carcinomas promote tumor growth and angiogenesis through elevated SDF-1/CXCL12 secretion. Cell 121: 335-348, 2005

10. De Wever O, Demetter P, Mareel M and Bracke M: Stromal myofibroblasts are drivers of invasive cancer growth. Int J Cancer 123: 2229-2238, 2008.

11. Tahara E: Growth factors and oncogenes in human gastrointestinal carcinomas. J Cancer Res Clin Oncol 116: 121-131, 1990.

12. Yashiro M, Chung YS and Sowa M: Role of orthotopic fibroblasts in the development of scirrhous gastric carcinoma. Jpn J Cancer Res 85: 883-886, 1994.
13. Japanese Gastric Cancer A: Japanese Classification of Gastric Carcinoma - 2nd English edition. Gastric Cancer 1: 10-24, 1998.

14. Maruyama M, Sasaki T and Kubota H: [Endoscopic treatment of early cancer of the large bowel]. Gan No Rinsho 32: 1309-1316, 1986.

15. Orimo A, Tomioka Y, Shimizu Y, et al: Cancer-associated myofibroblasts possess various factors to promote endometrial tumor progression. Clin Cancer Res 7: 3097-3105, 2001.

16. Nakazawa K, Yashiro M and Hirakawa K: Keratinocyte growth factor produced by gastric fibroblasts specifically stimulates proliferation of cancer cells from scirrhous gastric carcinoma. Cancer Res 63: 8848-8852, 2003.

17. Inoue $\mathrm{T}$, Chung YS, Yashiro $\mathrm{M}$, et al: Transforming growth factor-beta and hepatocyte growth factor produced by gastric fibroblasts stimulate the invasiveness of scirrhous gastric cancer cells. Jpn J Cancer Res 88: 152-159, 1997.

18. Tsujino T, Seshimo I, Yamamoto H, et al: Stromal myofibroblasts predict disease recurrence for colorectal cancer. Clin Cancer Res 13: 2082-2090, 2007.

19. Wipff PJ, Rifkin DB, Meister JJ and Hinz B: Myofibroblast contraction activates latent TGF-betal from the extracellular matrix. J Cell Biol 179: 1311-1323, 2007.

20. Ronnov-Jessen L and Petersen OW: Induction of alpha-smooth muscle actin by transforming growth factor-beta 1 in quiescent human breast gland fibroblasts. Implications for myofibroblast generation in breast neoplasia. Lab Invest 68: 696-707, 1993.

21. Saito H, Tsujitani S, Oka S, et al: The expression of transforming growth factor-betal is significantly correlated with the expression of vascular endothelial growth factor and poor prognosis of patients with advanced gastric carcinoma. Cancer 86: 1455-1462, 1999.

22. Kinugasa S, Abe S, Tachibana M, et al: Overexpression of transforming growth factor-betal in scirrhous carcinoma of the stomach correlates with decreased survival. Oncology 55: 582-587, 1998

23. Yoshida K, Yokozaki H, Niimoto M, Ito H, Ito $M$ and Tahara E: Expression of TGF-beta and procollagen type I and type III in human gastric carcinomas. Int J Cancer 44: 394-398, 1989. 